\title{
SÍNDROME DAS PERNAS INQUIETAS
}

\section{DIAGNÓSTICO E TRATAMENTO}

\section{Opinião de Especialistas Brasileiros}

\author{
Grupo Brasileiro de Estudos em Síndrome das Pernas Inquietas (GBE-SPI) ${ }^{1}$
}

\begin{abstract}
RESUMO - Este artigo contém as conclusões de reunião de 17-18 de novembro de 2006 do Grupo Brasileiro de Estudo em Síndrome das Pernas Inquietas (GBE-SPI) sobre diagnóstico e tratamento de SPI. Reiterou-se que se trata de condição de diagnóstico exclusivamente clínico, caracterizada por sensação anormal localizada, sobretudo, mas não exclusivamente, em membros inferiores, com piora noturna e alívio por movimentação da parte envolvida. Agentes terapêuticos com eficácia comprovada por estudos classe I são agonistas dopaminérgicos, levodopa e gabapentina enquanto que ácido valpróico de liberação lenta, clonazepam, oxicodona e reposição de ferro têm eficácia sugerida por estudos classe II. As recomendações do GBE-SPI para manejo de SPI primária são medidas de higiene do sono, suspensão de agentes agravantes de SPI, tratamento de comorbidades e agentes farmacológicos. Para estes as drogas de primeira escolha são agentes dopaminérgicos; segunda escolha são gabapentina ou oxicodona; e terceira escolha são clonazepam ou ácido valpróico de liberação lenta.
\end{abstract}

PALAVRAS-CHAVE: síndrome das pernas inquietas, movimentos periódicos das pernas, sono, pramipexole, levodopa, agonistas dopaminérgicos, gabapentina, oxicodona, clonazepam, ácido valpróico de liberação lenta.

\section{Restless legs syndrome: diagnosis and treatment. Opinion of Brazilian experts}

ABSTRACT - This article contains the conclusions of the November 17-18, 2006 meeting of the Brazilian Study Group of Restless Legs Syndrome (GBE-SPI) about diagnosis and management of restless legs syndrome (RLS). RLS is characterized by abnormal sensations mostly but not exclusively in the legs which worsen in the evening and are improved by motion of the affected body part. Its diagnosis is solely based on clinical findings. Therapeutic agents with efficacy supported by Class I studies are dopamine agonists, levodopa and gabapentine. Class II studies support the use of slow release valproic acid, clonazepan and oxycodone. The GBE-SPI recommendations for management of SPI are sleep hygiene, withdrawal of medications capable of worsening the condition, treatment of comorbidities and pharmacological agents. The first choice agents are dopaminergic drugs, second choice are gabapentine or oxycodone, and the third choice are clonazepan or slow release valproic acid.

KEY WORDS: restless legs syndrome, periodic leg movements, sleep, pramipexole, levodopa, dopamine agonists, gabapentine, oxycodone, clonazepam, slow release valproic acid.

\footnotetext{
${ }^{1}$ Membros do GBE-SPI: Flávio Aloe (Departamento de Neurologia, Hospital das Clínicas, USP-SP), Rosana S Cardoso Alves (Serviço de Neurologia Infantil, Departamento de Neurologia, Hospital das Clínicas, USP-SP), Luiz Augusto Franco Andrade (Instituto do Cérebro do Hospital Israelita Albert Einstein, São Paulo), Márcia Assis (prática privada, Curitiba, PR), Andrea Bacelar (prática privada, Rio de Janeiro, RJ), Márcio Bezerra (prática privada, Rio de Janeiro, RJ), Francisco Cardoso (Departamento de Clínica Médica, Setor de Neurologia, Universidade Federal de Minas Gerais)*, Henrique Ballalai Ferraz (Departamento de Neurologia, Escola Paulista de Medicina, UNIFESP), Ronaldo Guimarães Fonseca (Departamento de Neurologia, UNESP, Botucatu-SP), Wagner Horta (Setor de Neurologia, UFCE), Mônica Santoro Haddad (Departamento de Neurologia, Hospital das Clínicas, USP-SP), Rosa Hasan (Departamento de Neurologia, Hospital das Clínicas, USP-SP), James Pitágoras de Mattos (prática privada, Rio de Janeiro, RJ), Gilmar Prado (Departamento de Neurologia, Escola Paulista de Medicina, UNIFESP), Geraldo Rizzo (prática privada, Porto Alegre, RS)*, Nonato Rodrigues (Departamento de Neurologia, UNB), Ademir Batista Silva (Departamento de Neurologia, Escola Paulista de Medicina, UNIFESP), Delson José da Silva (Serviço de Neurologia, Hospital das Clínicas, UFG), Hélio Afonso Ghizoni Teive (Departamento de Clínica Médica, Setor de Neurologia, UFPR). *Responsáveis pela redação final do texto.
}

Recebido 28 Fevereiro 2007. Aceito 24 Abril 2007. 
A síndrome das pernas inquietas (SPI) foi inicialmente identificada em 1672 por Sir Thomas Willis, médico e anatomista inglês ${ }^{1}$ mas foi apenas em 1945 que o neurologista Karl Ekbom, na Suécia, redescobriu esta síndrome publicando um relato de caso ${ }^{2}$. Apesar desta longa história, apenas recentemente SPI tem despertado maior interesse na comunidade médica. Possivelmente, a maior razão para isto é a constatação que se trata de afecção neurológica bastante freqüente. Ainda que pareçam existirem diferenças étnicas na sua prevalência e não existam estudos epidemiológicos brasileiros, estimativas atuais apontam que pelo menos $5 \%$ da população sejam portadores de SPI ${ }^{3}$.

A característica fundamental desta enfermidade é a presença de desconforto, especialmente notável nas pernas (ainda que possa afetar outras regiões do corpo), cuja intensidade tem flutuação circadiana (aumenta à noite) e é aliviado por movimentação da parte corporal afetada. A maioria dos pacientes tem formas primárias de SPI, onde se reconhece a presença de significativa contribuição genética. No entanto, em cerca de $1 / 3$ dos casos SPI é secundária a outras condições, como neuropatias periféricas e outros ${ }^{4}$. Com o aumento do interesse nesta condição, vários estudos demonstram que existe amplo armamentário terapêutico capaz de aliviar seus sintomas.

O objetivo deste artigo é relatar as conclusões dos trabalhos da reunião do Grupo Brasileiro de Estudo da Síndrome das Pernas Inquietas (GBE-SPI), que se reuniu em São Paulo, SP, nos dias 17 e 18 de novembro de 2006 para discutir diagnóstico e tratamento da SPI nas condições de prática médica do Brasil.

\section{MÉTODO}

O GBE-SPI fez revisão sistemática da literatura publicada sobre diagnóstico e tratamento da SPI. Para isto, levantaram-se todos os artigos publicados e postados no Medline até novembro de 2006 relativos a este tema. Sua análise foi feita levando-se em conta os critérios sugeridos pela American Academy of Neurology e European Federation of Neurological Sciences ${ }^{5,6}$. Como será detalhado nos dois parágrafos seguintes, estes critérios podem levar à definição da utilidade ou inutilidade de métodos diagnósticos e terapêuticos. Este artigo, porém, descreve conclusões positivas (isto é, ocasiões quando o método diagnóstico é útil e a intervenção terapêutica é eficaz).

Para fins desta comunicação, foram selecionados estudos Classe I e II. No quesito diagnóstico, Classe I significa estudo prospectivo em amplo espectro de pessoas com a condição suspeita, usando um "padrão ouro" para a definição de caso, em que o teste é aplicado por meio de avaliação cega, de modo a permitir avaliação do teste apropriado para a acurácia diagnóstica. Por estudo de Classe II de diagnóstico compreende-se estudo prospectivo de pe- quena fração de pacientes, ou estudo bem desenhado, retrospectivo, de ampla quantidade de pessoas com a condição estabelecida (através de "padrão-ouro") comparadas a ampla quantidade de indivíduos controle, onde o teste é aplicado por meio de avaliação cega, de modo a permitir avaliação do teste apropriado para a acurácia diagnóstica. O uso de ao menos um estudo Classe I ou pelo menos dois estudos Classe II permite definir se um teste diagnóstico é ou não é útil ou preditivo do diagnóstico (recomendação Classe A). Adicionalmente, o GBE-SPI emite sua opinião baseada nas peculiaridades de prática médica no Brasil e na experiência pessoal de cada um de seus membros. Por fim, deve ser ressaltado que as recomendações deste texto, ainda que baseadas em criteriosa e sistemática revisão da literatura médica sobre o assunto e na atividade de experientes clínicos, não representam as únicas opções diagnóstica e terapêutica possíveis e, de forma alguma, têm implicações médico-legais.

Também para avaliação de métodos terapêuticos foram usados critérios Classe I e II. Um estudo qualifica para a primeira categoria se é ensaio clínico em população representativa, com poder estatístico adequado, prospectivo, randomizado e com avaliação cega de variável primária, ou é revisão sistemática com adequado poder estatístico de ensaios clínicos prospectivos, randomizados, em população representativa e com poder estatístico adequado. Estudos que qualificam para Classe I devem ter (A) randomização às cegas dos investigadores, (B) objetivo(s) primário(s) claramente definidos, (C) critérios de inclusão e exclusão claramente definidos, (D) contabilidade adequada de abandonos do estudo e mudança de braço de randomização ('cross-over') cujos números sejam baixos o suficiente para não criarem viés na análise, (E) apresentação clara de características de linha de base dos pacientes e que não difiram dos participantes do grupo controle. Já estudos são considerados Classe II sob o ponto de vista terapêutico se forem avaliação de coorte de pacientes e controle, prospectivo, em população representativa, com avaliação cega de variável primária e que tenham características $(A)$ a $(E)$ acima mencionadas ou se forem ensaio clínico prospectivo, controlado, em uma população representativa mas que não tenham uma das características (A) a (E) acima mencionadas. A partir de um estudo classe I ou pelo menos dois estudos classe II pode-se definir uma intervenção terapêutica como eficaz, ineficaz ou danosa (recomendação classe A).

\section{RESULTADOS \\ Diagnóstico}

O diagnóstico de SPI é exclusivamente clínico, obedecendo os critérios diagnósticos mínimos de acordo com o Grupo de Estudos Internacionais da Síndrome das Pernas Inquietas (International Restless Legs Syndrome Study Group - IRLSSG) ${ }^{7,8}$ e da Classificação Internacional dos Transtornos do Sono de 20059: (1) Necessidade irresistível e intensa de mover as pernas geralmente acompanhada de ou causada por sensações parestésicas desagradáveis nas pernas entre o tornozelo e o joelho. Por vezes a compulsão 
para movimentar os membros não é acompanhada do desconforto característico. Outros segmentos do corpo (braços) além das pernas podem ser acometidos, mas raramente desacompanhados do acometimento das pernas. A destacar que o profissional que cuida destes pacientes deve ficar atento às diversas expressões que podem ser usadas para caracterizar as queixas sensoriais: dor, ardor, dormência, desconforto, mal estar, peso, formigamento, sensação de inseto andando na perna etc. (2) A necessidade de mover as pernas ou a sensação desagradável de desconforto começa ou piora durante períodos de repouso ou inatividade, com o paciente sentado ou deitado. (3) A necessidade de mover as pernas ou a sensação desagradável é aliviada total ou parcialmente por movimentos tais como caminhar, alongar-se, curvar-se. Alguns pacientes sentem alívio com manobras sensitivas (banhos frios ou quentes, posturas específicas, massagens, etc). (4) A necessidade de mover as pernas ou a sensação desagradável apresenta uma característica circadiana surgindo ou piorando no final do dia e à noite. As queixas são geralmente mais leves durante o dia do que à noite e ocorrem picos de intensidade na madrugada.

Há, ainda, critérios de apoio ao diagnóstico: dados clínicos ou laboratoriais que complementam o diagnóstico em casos especiais ou duvidosos como, por exemplo, em pacientes demenciados ou em crianças que não podem fornecer um ou mais dos quatro critérios essenciais acima descritos. (1) Presença de movimentos periódicos de membros durante o sono (PLMS) e durante a vigília; (2) História familiar positiva de SPI, com hereditariedade sugestiva de ser autossômica dominante; (3) Resposta significativa com doses baixas de agentes dopaminérgicos.

\section{Diagnóstico de SPI na criança (2-12 anos)}

O diagnóstico de SPI na criança é feito pelo preenchimento dos quatro critérios diagnósticos dos adultos mais relato da própria criança consistente com a sensação desagradável e/ou desconforto nas pernas ou preenchimento dos 4 critérios diagnósticos dos adultos, sem o relato da criança, porém mais 2 dos seguintes critérios: (1) Transtorno de sono; (2) Familiar de primeiro grau portador de SPI; (3) Polissonografia com PLMI (índice de movimentos periódicos de membros) igual ou superior a 5 .

\section{SPI no paciente demenciado}

(1) Sinais sugestivos de desconforto nas pernas: esfregar, massagear, agachar, gemer manipulando as próprias pernas; (2) Atividade motora excessiva nos membros inferiores; (3) Sinais sugestivos de desconforto nas pernas que surgem ou pioram no repouso ou inatividade; (4) Sinais sugestivos de desconforto nas pernas melhoram com a atividade; (5) Os dois primeiros critérios ocorrem exclusivamente ou se intensificam ao entardecer ou à noite.

\section{Fatores de risco para SPI}

São considerados fatores de risco para o aparecimento de SPI as seguintes situações: (1) Sexo feminino; (2) Idade >50 anos; (3) Gravidez; (4) Deficiência de ferro; (5) Doação freqüente de sangue; (6) Insuficiência renal crônica; (7) Neuropatia periférica; (8) Uso excessivo de álcool ou bebidas cafeinadas; (9) Uso de fármacos como antidepressivos, antihistamínicos, metoclopramida, lítio etc.; (10) Outros: doença de Parkinson, diabetes mellitus, fibromialgia, artrite reumatóide, mielopatias, transtornos do sono como narcolepsia e distúrbio comportamental do sono REM.

\section{Diagnóstico diferencial}

Existem várias condições que lembram a SPI, mas os dados de história familiar, a caracterização de sintomas circadianos e a insônia auxiliam substancialmente no diagnóstico diferencial. Adiante seguem algumas condições que podem ser confundidas com SPI:

Neuropatia periférica - Polineuropatia periférica do tipo sensitiva (PNPS) e radiculopatia produzem sintomas de queimação e dor na superfície da pele, ao contrário da SPI que produz sensação na profundidade da musculatura. Os sintomas podem piorar a noite, mas estão presentes durante o dia, não meIhoram com atividade motora ou mental e não há PLMS. O exame neurológico é anormal com redução dos reflexos tendíneos profundos A PNPS pode, entretanto, estar associada com SPI, tornando o diagnóstico diferencial extremamente difícil. Nesse caso está indicada a realização de eletroneuromiografia.

Acatisia - A acatisia está relacionada ao uso de drogas que bloqueiam os receptores da dopamina, como antipsicóticos e metoclopramina e com outros sintomas extra-piramidais como tremores e discinesia facial. Nestes casos, a necessidade de movimentação não é acompanhada por parestesias, as quais não se restringem aos membros inferiores, mas sim ao corpo todo; a inquietação motora é caracterizada por marchar no mesmo lugar e andar de um lado para o outro, não há tendência em piorar à noite ou em repouso. 
Insuficiência vascular periférica - A dor piora com a movimentação e melhora com o repouso, há alterações locais na pele, veias varicosas, e não há história familiar e alterações do sono.

Síndrome das pernas dolorosas com movimentos dos artelhos - Esta condição incomum (comumente conhecida pelo nome em inglês painful legs and moving toes) relaciona-se freqüentemente a trauma periférico dos membros inferiores e se caracteriza dor difusa e intensa nos pés ou pernas, acompanhada por movimentos de flexão ou extensão dos artelhos antes sono. Estes movimentos, no entanto, são involuntários, freqüentemente desaparecem durante o sono, não necessariamente pioram com o repouso e não melhoram com atividade.

Cãibras noturnas - As cãibras noturnas das pernas melhoram com alongamento, contudo ocorrem contrações palpáveis nos músculos afetados, com localização fixa e ou unilateral e não há desconforto sensorial.

Transtorno do déficit de atenção e hiperatividade (TDAH) - Uma condição especialmente importante para o diagnóstico diferencial é o TDAH na população pediátrica escolar ${ }^{10}$. Um dos critérios diagnósticos de TDAH pelo DSM-IV é a presença de movimentação excessiva de mãos e pés, levantar-se na sala de aula e sensação de inquietação motora que pode ser facilmente confundida com os critérios diagnósticos números 1 e 2 de SPI. Crianças com SPI apresentam irritabilidade, inquietude motora na sala de aula por causa do desconforto físico relacionado com a SPI que pode ser facilmente interpretado como hiperatividade, desatenção ou causar desatenção. Além disso, crianças com TDAH apresentam alterações do sono com PLMS que podem ser confundidas com SPI.

Outros diagnósticos diferenciais - A lista de condições que podem ser confundidas com SPI ainda inclui acatisia hipotensiva, estados de ansiedade, delírio de infestação dos membros, estados de excitação maníaca, tremor ortostático, desconforto posicional, flutuações motoras no paciente parkinsoniano, dor do crescimento, mioclonias noturnas, distúrbio rítmico do movimento relacionado ao sono, estereotipias (balançar as pernas ou pés), tremor ortostático e PLMS sem SPI.

Exames complementares - A investigação de alterações do metabolismo de ferro com dosagem sé- rica de ferro, níveis de ferritina e saturação da transferrina deve ser realizada em todos os pacientes. A dosagem de ferritina e transferrina sérica devem ser idealmente realizadas por volta das 21 horas. A ferritina no líquor $(<6,5 \mu / \mathrm{L}(\mathrm{ng} / \mathrm{mL}))$ é mais específica e sensível do que a ferritina sérica isoladamente e a retirada do LCR deve ser realizada por volta das 22 horas. Outros marcadores bioquímicos importantes são: glicemia, creatinina, TSH, teste de gravidez, dosagem de cálcio e magnésio, vitamina B12 e ácido fólico. A eletroneuromiografia deve ser solicitada quando houver suspeita de neuropatia periférica. A polissonografia (PSG) não é rotineiramente indicada para o diagnóstico de SPI. Contudo, quando o diagnóstico for duvidoso, quando o quadro clínico diurno for leve e houver sintomas intensos de fragmentação de sono, quando houver suspeita de síndrome da apnéia obstrutiva do sono simultaneamente e se houver persistência de sintomas de fragmentação do sono mesmo após o tratamento, a polissonografia pode ser muito útil. Outro teste de auxilio diagnóstico é a actigrafia que registra períodos de atividade e inatividade de membros inferiores. $\mathrm{O}$ teste da imobilização forçada, proposto por Montplaisir et al. ${ }^{11}$, é uma manobra de provocação dos sintomas de SPI através da imobilização do pacientes. É usado quase que exclusivamente no contexto de investigação científica. Por fim, recentemente foi feita a validação do teste de levodopa, que consiste na administração de uma dose de $100 \mathrm{mg}$ de levodopa com $25 \mathrm{mg}$ de benserazida no horário de inicio dos sintomas sensoriais com subseqüente registro da intensidade desses sintomas sensoriais a cada 15 minutos por um período total de 2 horas $^{12}$.

\section{Aumentação ("Augmentation")}

A aumentação é uma intercorrência clínicamente significativa e exclusiva do tratamento da SPI e geralmente aparece nos primeiros seis meses mas pode se manifestar a qualquer momento do tratamento ${ }^{13,14}$. Ocorre mais precocemente com levodopa e é geralmente mais branda e tardia com agonistas dopaminérgicos. Entre os fatores de risco para a ocorrência de aumentação estão o uso de doses acima de 200 mg de levodopa, formas secundárias da SPI, ferritina sérica e liquórica baixa, perda crônica de ferro e o nível de gravidade do quadro clínico pré-tratamento como nas formas diárias e refratárias. Sexo e idade não representam riscos de aumentação. Não há relatos de aumentação com antiepilépticos ou opióides (exceção ao tramadol). A aumentação é portanto um fenômeno dependente do agente farmacoló- 
gico dopaminérgico, quer seja precursor ou agonista dopaminérgico ${ }^{15}$.

O diagnóstico da aumentação de acordo com os critérios diagnósticos IRLSSG são os seguintes ${ }^{13,15}$ : 0 quadro clínico deve ter pelo menos cinco dias seguidos, na ausência de transtornos psiquiátricos, médicos ou drogas que justifiquem o seguinte: (1) Sintomas sensitivo-motores que passam a ocorrer duas horas ou menos do que o horário indicado pelo paciente e eventualmente há desaparecimento das características circadianas (expansão temporal). Adiantamento do horário dos sintomas é o sintoma mais freqüente e confiável de expansão e ocorre em cerca de $96 \%$ dos casos (93); (2) Piora dos sintomas com aumento da dose de levodopa ou agonistas dopaminérgicos e melhora com a diminuição dessas drogas. A piora dos sintomas pode atingir níveis acima daqueles pré-tratamento.

Aumentação, rebote, tolerância e progressão da SPI são fenômenos que devem ser diferenciados clinicamente por causa das suas conseqüências na decisão terapêutica. Rebote ( $25 \%$ ) é o reaparecimento e intensificação de sintomas após o final do efeito da dose. Tolerância é a perda do efeito terapêutico de certa dose de medicamento. Aumento da dose produz eliminação dos sintomas de tolerância, exatamente o contrário do que acontece na aumentação. Progressão é aumento lento e progressivo dos sintomas sem expansão temporal ou expansão espacial em pacientes que estão em uso de agonistas dopaminérgicos, precursores dopaminérgicos, opióides, anti-epilépticos ou outras classes de medicações.

\section{Terapêutica}

Agentes dopaminérgicos - A levodopa é uma pródroga que se transforma em dopamina pela ação de descarboxilase de aminoácidos aromáticos. Estudo Classe $\mathrm{I}^{16}$ mostra que este agente tem eficácia definida para o tratamento da SP. Os efeitos colaterais mais comuns são náusea, vômito, cefaléia, hipotensão postural, sonolência. $O$ fenômeno de aumentação tem sido relatado em média em $60 \%$ dos pacientes tratados com este agente (mínimo de $30 \%$ e máximo de $80 \%$ ) enquanto que 20 a $35 \%$ (média de $25 \%$ ) dos portadores de SPI expostos a levodopa desenvolvem rebote. A combinação de levodopa de liberação regular com a de liberação lenta transitoriamente reduz as manifestações de aumentação que recorrem em $60 \%$ dos pacientes ${ }^{17,18}$. A experiência clínica indica que esta droga é eficaz em todas formas clínicas e na maioria dos pacientes mas sua eficiência é limitada por rápido aparecimento de efei- tos colaterais. Recomenda-se uso de no máximo 200 $\mathrm{mg}$ ao dia, que deve ser administrado sob forma de dose única 30-60 min antes de hora de aparecimento habitual de sintomas para forma regular e 60-120 min para forma lenta. Pode ser considerado uso intermitente e é agente particularmente seguro para uso durante a gravidez. Cautela maior quando ferritina/ferro séricos estiverem baixos, pelo risco elevado de aumentação.

Agonistas dopaminérgicos diretos agem diretamente no receptor dopaminérgico, dispensando sua prévia metabolização. Pergolida, cabergolina e rotigotina são exemplos destas drogas da geração mais antiga de drogas derivadas do ergot, os agonistas ergolínicos. Mais recentemente, foram desenvolvidas drogas não relacionadas com o ergot, os chamados agonistas não-ergolínicos, dentre os quais se salientam pramipexole e ropinirole. Estudos Classe I e II para cabergolina, pergolida, pramipexole, ropinirole e rotigotina ${ }^{19-23}$ mostram que estes agentes têm eficácia definida no tratamento de SPI. Os efeitos colaterais mais comuns são náusea, vômito, cefaléia, hipotensão postural, sonolência, edema, alucinação. Raramente, agentes ergolínicos podem causar fibrose pericárdica, valvular, pleural e retroperitoneal. Ainda que existam relativamente poucos estudos para os diferentes membros desta categoria de drogas, há relatos de aumentação ocorrendo em 3 a 37\% dos pacientes com SPI em tratamento com agonistas. Desconhece-se a freqüência de aparecimento de rebote. Não existem estudos comparativos para avaliar eventuais diferenças entre os diversos agonistas dopaminérgicos. A experiência clínica mostra que agonistas são eficazes e bem tolerados em todas as formas clínicas na maioria dos pacientes. Recomenda-se que estes agentes sejam administrados em dose única diária $2 \mathrm{~h}$ antes do início habitual dos sintomas. As doses recomendadas para os diferentes agentes são: bromocriptina - 2,5 a 7,5 mg; cabergolina - 0,5 a 2,0 mg; pergolida - 0,05 a 0,5 mg; pramipexole $-0,125$ a $0,750 \mathrm{mg}$; ropinirole $-0,25$ a $1,0 \mathrm{mg}$; rotigotina adesivo de 4 a $6 \mathrm{mg}^{18}$.

Anticonvulsivantes - Um estudo Classe I mostra que a gabapentina é definidamente eficaz para o tratamento da $\mathrm{SP}^{24}$. Os efeitos colaterais mais comuns neste estudo foram indisposição geral, sonolência e sintomas gastrointestinais. A experiência clínica mostra que este agente é particularmente útil para SPI secundária a neuropatias periféricas com sintomas álgicos. A dose eficaz para o alívio de sintomas de SPI é costumeiramente alta, na ordem de $1800 \mathrm{mg}$ 
ao dia (administrada $2 \mathrm{~h}$ antes do horário de início habitual de sintomas), que deve ser alcançada gradual e lentamente ${ }^{18,24}$.

O outro anticonvulsivante comprovadamente eficaz no tratamento de SPI é ácido valpróico de liberação lenta, que foi testado através de estudo Classe $11^{25}$. O principal efeito colateral nestes ensaios foi sonolência, com dose recomendada de $300 \mathrm{mg}$ iniciais, elevada para $600 \mathrm{mg}$ dois dias mais tarde, com administração única e $2 \mathrm{~h}$ antes do horário de início habitual de sintomas ${ }^{18}$. Não pode ser esquecida a necessidade de monitoração periódica da função hepática. De ressalva, nenhum dos membros do GBESPI tem experiência pessoal com o uso desta droga no manejo de SPI.

Benzodiazepínicos - O único benzodiazepínico que satisfaz os critérios metodológicos da presente análise sistemática é o clonazepam. Um estudo Classe II indica que este agente é possivelmente eficaz para o manejo de $\mathrm{SP}^{26}$. A ressaltar, porém, que este estudo apenas incluiu seis pacientes e que um outro ensaio Classe II não confirmou a eficácia do clonazepam para $\mathrm{SP}^{27}$. O efeito colateral mais comumente observado é sonolência diurna. A dose diária recomendada, administrada em única tomada ao deitar, é 0,2 a 1,0 mg ${ }^{18}$. A experiência clínica do GBE-SPI mostra que se trata de agente particularmente útil em pacientes com ansiedade, fragmentação e privação do sono. Vale ressaltar que inexistem evidências científicas Classes I e II quanto à eficácia e segurança de outros benzodiazepínicos e agentes hipnóticos não-benzodiazepínicos em SPI.

Opióides - Estudo Classe II indica que a oxicodona tem eficácia definida no tratamento farmacológico da $\mathrm{SPI}^{28}$. Os efeitos colaterais mais comuns são constipação, letargia diurna e distúrbios respiratórios noturnos. A dose média recomendada de oxicodona é $10 \mathrm{mg}$ (podendo variar de 5 a $20 \mathrm{mg}$ ), administrada em dose única, $2 \mathrm{~h}$ antes do início habitual dos sintomas $^{18}$. A experiência clínica sugere que oxicodona é particularmente útil para pacientes com formas de SPI refratárias e/ou com desenvolvimento de aumentação ao serem tratados com outros agentes. Precaução adicional é requerida em pacientes com histórico de droga-adição. Para outros opióides (codeína, metadona, morfina, propoxifeno e tramadol) apenas existem estudos Classes III e IV.

Reposição de ferro - Estudos Classe II mostram que reposição endovenosa de ferro tem eficácia de- finida no tratamento de SPI associada a IRC ${ }^{29}$. Por outro lado, ferro administrado oralmente é ineficaz para o tratamento da SPI primária conforme estudo Classe ${ }^{30}$. No estudo de administração parenteral deste agente, os efeitos colaterais mais comuns foram náusea, constipação, mudança de cor de dentes, fratura de vértebra, fezes escurecidas e piora da SPI. Apesar da escassez de dados na literatura, os membros do GBE-SPI relatam que em sua experiência clínica ferro oral pode ser útil em pacientes com SPI no contexto de anemia ferropriva, gestação, com ferritina baixa e IRC. A dose recomendada de ferro iônico é 4-6 mg/kg/dia (100 mg de ferro iônico em $500 \mathrm{mg}$ de sulfato ferroso), associado à vitamina $C$, fora das refeições e em dose única diária. A duração do tratamento depende da condição de base.

\section{CONCLUSÃO}

A análise sistemática da literatura feita pelo GBESPI confirma a noção amplamente estabelecida que o diagnóstico de SPI é feito com bases exclusivamente clínicas, não havendo necessidade de propedêutica laboratorial. Ponto ressaltado pelos especialistas participantes do encontro é a necessidade de atenção à diversidade de expressões que são utilizadas pelo brasileiro para descrever o desconforto associado à SPI. Adiante seguem recomendações terapêuticas para manejo de SPI em diferentes situações:

\section{SPI primária - Agentes farmacológicos}

$1^{\text {a }}$ Escolha: Agentes dopaminérgicos (cabergolina, pergolida, pramipexole, ropinirole, levodopa). Não há evidências que favoreçam um agonista sobre o outro. Levodopa tem maior potencial de causar aumentação e rebote.

$2^{\text {a }}$ Escolha: Gabapentina ou oxicodona.

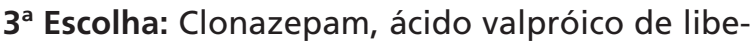
ração lenta; combinações.

Outras medidas: Higiene do sono; suspensão de fármacos agravantes (agentes antidopaminérgicos, inibidores super-seletivos da recaptação de serotonina, tricíclicos, lítio, antihistamínicos, melatonina etc); evitar consumo de produtos ricos em cafeína, bebida alcóolica e exercício físico intenso antes de dormir; tratar comorbidades (outros transtornos do sono etc).

\section{SPI secundária}

Tratamento da causa quando possível. Há medidas apropriadas a situações específicas:

Gravidez: Levodopa, suplementação de ferro.

Insuficiência renal crônica dialítica: Suplementa- 
ção de ferro. Relatos de caso de cura pós-transplante renal.

Neuropatia periférica: Gabapentina.

\section{Aumentação}

É importante frisar que os estudos relativos a esta situação são todos Classe IV ${ }^{15}$. No entanto, trata-se de problema de tal relevância clínica que os membros do GBE-SPI decidiram-se por emitir as recomendações que se seguem: não aumentar a dose de agentes dopaminérgicos (a aparente redução de eficácia terapêutica comumente induz o médico a aumentar a dose da medicação o que, comprovadamente, piora a aumentação); antecipar a dose para $2 \mathrm{~h}$ antes do novo início dos sintomas; alternar diferentes agentes dopaminérgicos entre si; trocar agentes dopaminérgicos por opióides e/ou gabapentina; utilizar associações de agentes de diferentes classes farmacológicas; sempre dosar ferritina e ferro séricos.

\section{Outras situações}

Inexistem dados para orientar de modo científico as escolhas terapêuticas em SPI em crianças. Opiniões de especialistas do GBE-SPI (isto é, evidência Classe IV) sugerem que ác. valpróico, carbamazepina, clonazepam, clonidina, levodopa além de medidas de higiene do sono podem ser úteis neste contexto. Também não há evidências Classes I e II para sustentar escolhas no manejo de depressão associada à SPI. Sugestões do GBE-SPI de drogas antidepressivas que não costumam piorar SPI são bupropiona, reboxetina e trazodona.

Agradecimento - Os membros do GBE-SPI agradecem ao laboratório Boehringer-Ingelheim pelo apoio que viabilizou a reunião de 17-18 de novembro de 2007 através de ajuda financeira sem restrições.

\section{REFERÊNCIAS}

1. Willis T. The London Practice of Physice, 1.Ed. London: Thomas Bassett and William Crooke, 1685:404.

2. Ekbom KA. Restless legs. Acta Med Scand 1945;158(Suppl):S4-S124.

3. Wayne H, Walters AS, Allen RP, et al. Impact, diagnosis and treatment of restless legs syndrome (RLS) in a primary care population: the REST (RLS epidemiology, symptoms, and treatment) primary care study. Sleep Medicine 2004;5:237-246.

4. Trenkwalder C, Paulus W, Walters AS. The restless legs syndrome. Lancet Neurol 2005;4:465-475.

5. Miller R, Edlund W. Process for developing practice parameters. 1999 AAN Quality Standards Subcommittee.

6. Brainin M, Barnes M, Baron JC, et al. Guidance for the preparation of neurological management guidelines by EFNS scientific task forces: revised recommendations 2004. Eur J Neurol 2004;11:577-581.

7. Walters AS. Toward a better definition of the restless legs syndrome. The International Restless Legs Syndrome Study Group. Mov Disord 1995;10:634-642.

8. American Academy of Sleep Medicine. Periodic limb movement disorder. International Classification of Sleep Disorders. Diagnostic and Coding Manual, 2nd edn. Westchester, IL: American Academy of Sleep Medicine, 2005:182-186.
9. American Academy of Sleep Medicine. Restless legs syndrome. International Classification of Sleep Disorders. Diagnostic and Coding Manual, 2.Ed. Westchester, IL: American Academy of Sleep Medicine, 2005: 178-181.

10. Walters AS, Picchietti DL, Ehrenberg BL, Wagner ML. Restless legs syndrome in childhood and adolescence. Pediatr Neurol 1994;11:241-245.

11. Montplaisir J, Boucher S, Nicolas A, et al. Immobilization tests and periodic leg movements in sleep for the diagnosis of restless leg syndrome. Mov Disord 1998;13:324-329.

12. Stiasny-Kolster K, Kohnen R, Moller JC, et al. Validation of the "L-DOPA test" for diagnosis of restless legs syndrome. Mov Disord 2006;21:1333 1339.

13. Chesson AL, Wise M, Davila D, et al. Practice parameters for the treatment of restless legs syndrome and periodic limb movement disorder: an American Academy Of Sleep Medicine Report. Standards of Practice Committee of the Ameriocan Academy of Sleep Medicine. Sleep 1999;22:961-988.

14. Allen RP, Picchietti D, Hening WA, Trenkwalder C, Walters AS, Montplaisir J. Restless legs syndrome: diagnostic criteria, special considerations, and epidemiology: a report from the restless legs syndrome diagnosis and epidemiology workshop at the National Institutes of Health Sleep Medicine 2003;4:101-119.

15. Paulus W, Trenkwalder C. Less is more: pathophysiology of dopaminergic-therapy-related augmentation in restless legs syndrome. Lancet Neurol 2006;5:878-886.

16. Benes H, Kurella B, Kummer J, et al. Rapid onset of action of levodopa in restless legs syndrome: a double-blind, randomized, multicenter, crossover trial. Sleep 1999;22:1073-1081.

17. Trenkwalder C, Collado Seidel V, Kazenwadel J, et al. One-year treatment with standard and sustained-release levodopa: appropriatelong-term treatment of restless legs syndrome? Mov Disord 2003;18:1184-1189.

18. Vignatelli L, Billiard M, Clarenbach P, et al. EFNS guidelines on management of restless legs syndrome and periodic limb movement disorder in sleep. Eur J Neurol 2006;13:1049-1065.

19. Stiasny-Kolster K, Benes H, Peglau I, et al. Effective cabergoline treatment in idiopathic restless legs syndrome. Neurology 2004;63:22722279.

20. Trenkwalder C, Hundemer HP, Lledo A, et al. Efficacy of pergolide in treatment of restless legs syndrome: the PEARLS Study. Neurology 2004;62:1391-1397.

21. Trenkwalder C, Garcia-Borreguero D, Montagna P, et al. Ropinirole in the treatment of restless legs syndrome: results from the TREAT RLS 1 study, a 12 week, randomised, placebo controlled study in 10 European countries. J Neurol Neurosurg Psychiatry 2004;75:92-97.

22. Montplaisir J, Nicolas A, Denesle R, Gomez-Mancilla B. Restless legs syndrome improved by pramipexole: a double-blind randomized trial. Neurology 1999;52:938-943.

23. Stiasny-Kolster K, Kohnen R, Schollmayer E, et al. Patch application of the dopamine agonist rotigotine to patients with moderate to advanced stages of restless legs syndrome: a double-blind, placebo-controlled pilot study. Mov Disord 2004;19:1432-1438.

24. Garcia-Borreguero D, Larrosa O, de la Llave Y, et al. Treatment of restless legs syndrome with gabapentin: a double-blind, cross-over study. Neurology 2002;59:1573-1579.

25. Eisensehr I, Ehrenberg BL, Rogge Solti S, Noachtar S. Treatment of idiopathic restless legs syndrome (RLS) with slow-release valproic acid compared with slow-release levodopa/benserazid. J Neurol 2004;251:579-583.

26. Montagna P, Sassoli de Bianchi L, Zucconi M, Cirignotta F, Lugaresi E. Clonazepam and vibration in restless legs syndrome. Acta Neurol Scand 1984;69:428-430.

27. Boghen D, Lamothe L, Elie R, Godbout R, Montplaisir J. The treatment of the restless legs syndrome with clonazepam: a prospective controlled study. Can J Neurol Sci 1986;13:245-247.

28. Walters AS, Wagner ML, Hening WA, et al. Successful treatment of the idiopathic restless legs syndrome in a randomized double-blind trial of oxycodone versus placebo. Sleep 1993;16:327-332.

29. Sloand JA, Shelly MA, Feigin A, Bernstein P, Monk RD. A double-blind, placebo-controlled trial of intravenous iron dextran therapy in patients with ESRD and restless legs syndrome. Am J Kidney Dis 2004;43: 663-670.

30. Davis BJ, Rajput A, Rajput ML, Aul EA, Eichhorn GR. A randomized, double-blind placebo-controlled trial of iron in restless legs syndrome. Eur Neurol 2000;43:70-75. 